\title{
Social choice among complex objects
}

\author{
Luigi Marengo And Simona Settepanella
}

\begin{abstract}
We present a geometric model of social choice when the latter takes place among bundles of interdependent elements, that we will call objects. We show that the outcome of the social choice process is highly dependent on the way these bundles are formed. By bundling and unbundling the same set of constituent elements an authority enjoys a vast power of determining the social outcome. We provide necessary and sufficient conditions under which a social outcome may be a local or global optimum for a set of objects, and we show that, by appropriately redefining the set of objects, intransitive cycles may be broken and the median voter may be turned into a loser.
\end{abstract}

Mathematics Subject Classification (2010): 05C20 (primary); 52C35 (secondary).

\section{Introduction}

Social choice theory usually assumes that agents are faced with a set of exogenously given and mutually exclusive alternatives. These alternatives are given in the sense that the pre-choice process through which they are constructed is not analyzed. Moreover, these alternatives are "simple", in the sense that they are one-dimensional objects or, even when they are multidimensional, they are simply points in some portion of the homogeneous $\mathbb{R}^{n}$ space and they lack an internal structure that limits the set of possible alternatives.

Many choices in real life situations depart substantially from this simple setting. Choices are often made among bundles of interdependent elements. Those bundles may be formed in a variety of ways, which in turn affect the selection process of a social outcome. Let us consider, for instance, a typical textbook example of social choice, i.e., the case of a group of friends deciding how to spend the evening by democratic pairwise majority voting. The textbook would start from a given and predefined choice set as $X=\{A, B, C, D, \ldots\}$ where the set $\{A, B, C, D, \ldots\}$ could stand for movie, concert, restaurant, dinner at home .... At closer scrutiny, these alternatives are neither primitive nor exogenously given. Going to the movies or to a restaurant are labels for bundles of elements (e.g., with whom, where, when, 
movie genre, director, type of food, etc.) and everyone's preference is unlikely to be expressed before these labels are specified in their constituting elements. A skillful member of the group could easily obtain a social outcome close to the one he or she prefers by carefully crafting the objects $A, B, C, D, \ldots$ and possibly by designing a new set of objects.

Moving on, to more serious examples, candidates and parties in political elections stand for complex bundles of interdependent policies and personality traits. Committees and boards are called upon to decide upon packages of policies, e.g., a recruitment package that a university governing board has to approve. In principle, any combination of elements (subject to a budget or some other constraint) could be considered and compared (e.g., through majority voting) with any other, but in reality only a relatively small number of packages undergo examination. Typically, the bundling of elements serves the purpose of reducing the number of alternatives to be examined, by decomposing the whole space of alternatives into smaller subspaces.

In this paper we present a geometric model of social choice among bundles of elements, which we call objects. Geometric approaches have already been used in the literature on social choice. Donald Saari [20-22] has greatly contributed to establishing general geometric representations of voting models and voting paradoxes and we will argue later that our representation is more general in many respects. The papers $[4,5,9,10]$ study the problem of the existence of a social decision function from a topological point of view and show that the paradoxes of social choice are partly a consequence of the topological structure of the spaces of ordinal preferences. On the other hand, [2] discusses the possibility of introducing topological methods in the combinatorial paradigm of social choice theory. The articles [28] and [27] extend well-known results on social choice functions to, respectively, CW complexes and arrangements, thus obtaining new results for both mathematical objects. In this respect, our model is a novel contribution to the analysis of discrete problems of social choice.

We deal with the issue of object construction that has not been dealt with by economic models. The main idea is that, first, generally objects are not simply aggregations of primitive components but have an internal structure that is likely to determine interdependencies and non-separabilities in individual preferences. In the "what shall we do tonight" choice setting, my preferences on the with whom element is likely to be highly interdependent with the other elements, as I may well find a given person a perfect companion for an evening at the movies but relatively dislike her or his company if we finally decide to go to a restaurant. Second, objects provide structure to the choice problem. Consider again the "what shall we do tonight" case. A possible reply to our point on bundles would be that the choice set $X$ is underspecified and that we should start from a choice set formed by all possible combinations of the elements. However, for obvious combinatorial arguments, this set, even in this simple example, would be so large that any exhaustive choice procedure, e.g., pairwise majority voting, could not be completed in a feasible time span. In our approach, objects decompose the search space into quasiseparable subspaces [26] and simplify the computational task, making decisions possible. 
There is also another way objects can contribute to making the determination of a social outcome easier. We will show that, by appropriate object construction, intransitive cycles that often characterize social decisions can almost always be eliminated. In general, coarse objects, i.e., those made of many elements, tend to produce many cycles, whereas fine objects do not. However finer objects do so by increasing the number of locally stable social optima and thereby making the social outcome more manipulable through the control of object construction, initial conditions and agendas. All in all, we will analyze how different sets of objects strike different balances in the trade-off between decidability and non-manipulability.

Our work is closely linked to the literature on agenda power [16,19], and we will show that we generalize some of its results in the sense that even agenda power is subject to manipulation through object design. It is also related to recent literature on multidimensional voting models $[7,11,13,25,25]$ and the one that has begun to analyze decision-making when agents group states of the world into coarse categories $[12,17]$, but there are fundamental differences that make our model new.

In order to formally analyze the properties of a social choice model with object construction power we use the tools of hyperplane arrangements theory and graph theory. Thanks to them, we show that the way objects are constructed by bundling (or unbundling) elements can strongly impact on the outcome of social choice. We show that, in general, by appropriately constructing objects, the outcome of a social choice process, e.g., pairwise majority voting, may be heavily manipulated. An authority who has the power to construct objects may obtain a desired outcome even when the latter is chosen democratically. We will prove necessary and sufficient conditions for any social outcome to be a locally or globally stable social optimum for a social decision rule under a specific set of objects. We will also show an algorithmic procedure to determine which set of objects, agendas and initial conditions can lead society, through a given social choice procedure, to select a given outcome. Moreover, the object construction power that we describe and analyze in this paper will be proven to be stronger and more general than the well-known agenda power in the sense that also the agenda power may be manipulated by appropriate object construction. Finally, we will discuss how another classic result, i.e., the so-called median voter theorem, may be reverted by appropriate modification of the set of objects, thus transforming the median voter into an outright loser.

This paper is structured as follows: in Section 2 we outline our geometric model. A key ingredient of our analytic approach is the theory of hyperplane arrangements. Then, in Section 3 we draw the main results concerning how, through object construction, it is possible to manipulate social outcomes, create or eliminate agenda power, create or eliminate intransitive cycles, and turn the median voter into a loser. We will also demonstrate that our object construction power is stronger and more general than the traditionally considered agenda power and that objects strike a balance in the trade-off between decidability and non-manipulability. In a companion paper [14] many analytical results obtained here are illustrated through examples and computational models. The interested reader can refer to it for algorithmic implementations of most of the arguments contained in the present paper. 
ACKnowledgements. We are greatly indebted to Corrado Pasquali, Mario Salvetti and Gennaro Amendola for sharing and discussing many of the ideas contained in this paper. We gratefully acknowledge the support given to this research by the European Commission, within the 6th FP Network of Excellence "DIME - Dynamics of Institutions and Markets in Europe" and the Specific Targeted Research Project "CO3 - Common Complex Collective Phenomena in Statistical Mechanics, Society, Economics and Biology". The usual caveat applies.

\section{Definitions and structure of the model}

Features We assume that choices are made over bundles of elements that we call features. The number of features is finite and each feature may take one value out of a finite set of alternatives. We call $F=\left\{f_{1}, \ldots, f_{n}\right\}$ the set of such features. In order to simplify the notation and without loss of generality, we assume that all features may take the same number $m+1$ of values in the set $\{0,1,2, \ldots, m\}$ $\forall i=1, \ldots, n$.

The space of social outcomes is given by all the $n$-tuples specifying one value for every feature; of course there are $(m+1)^{n}$ of them. We call $X$ the set of all possible social outcomes and $x=\left(v_{1} . \cdots . v_{n}\right)$ a generic element thereof.

Example 2.1. Let us consider an example of 3 features $\left\{f_{1}, f_{2}, f_{3}\right\}$ taking a value out of the binary set $\{0,1\}$. This is equivalent to, e.g., the case of presence or absence of three possible traits. Thus the space $X$ of possible social outcomes is a set of 8 ordered 3-tuples of the form $x=\left(v_{1} \cdot v_{2} \cdot v_{3}\right)$ for $v_{i} \in\{0,1\}, i=1,2,3$. This can be regarded as the example in Introduction: a group of friends deciding how to spend the evening. Hence the 3 features could be \{where to go, when to go, how to go $\}$ taking a value in $\{0,1\}, e . g$., where to go \{restaurant, movies\}, when to go $\{8 \mathrm{pm}, 10 \mathrm{pm}\}$, how to go by car, walking . An element $x=\left(v_{1} \cdot v_{2} . v_{3}\right)$ in $X$ would be, for example, (restaurant.8pm.car).

The hyperplane arrangement Given a set of features $F=\left\{f_{1}, \ldots, f_{n}\right\}$ taking values in the set $\{0,1,2, \ldots, m-1\}$, we can associate to it the hyperplane arrangement $^{1}$ in $\mathbb{R}^{n}$

$$
\mathcal{A}_{n, m}=\left\{H_{i, j} \mid \alpha_{H_{i, j}}=\lambda_{i}-j\right\}_{\substack{1 \leqslant i \leqslant n \\ 0 \leqslant j<m-1}}
$$

where the indices $1 \leqslant i \leqslant n$ and $0 \leqslant j<m-1$ vary, respectively, among the indices of features and the indices of values taken by features.

The social outcome $x=\left(v_{1}, \cdots . v_{n}\right)$ will correspond to the chamber $C_{x}$ that contains the open set

$$
\left\{\left(\lambda_{1}, \ldots, \lambda_{n}\right) \in \mathbb{R}^{n} \mid v_{j}-1<\lambda_{j}<v_{j}, j=1, \ldots, n\right\} .
$$

${ }^{1}$ For the reader who is unfamiliar with hyperplane arrangements, some basic notions are provided in [24]. 
Let us remark that in our framework the number $n$ of features gives the dimension of the space $\mathbb{R}^{n}$ and the values $v_{i} \in\{0,1, \ldots, m-1\}$ taken by the feature $f_{i}$ provide a set of parallel hyperplanes.

Example 2.2. In Example 2.1 the associated arrangement $\mathcal{A}_{3,2}$ is simply the arrangement given by the coordinate hyperplanes of an orthogonal Cartesian system in $\mathbb{R}^{3}$. Hence we get 8 chambers corresponding to the 8 different octants in which the orthogonal Cartesian system divides $\mathbb{R}^{3}$. Each social outcome $x \in X$ corresponds to an octant $C_{x}$.

Let us remark that, in general, there is no relation between the $n$-tuple $x=$ $\left(v_{1}, \cdots . v_{n}\right)$ and the point $\left(v_{1}, \ldots, v_{n}\right) \in \mathbb{R}^{n}$. Nevertheless, depending on what we are choosing upon (e.g., if features take values on real numbers), we can slightly modify the arrangement of hyperplanes defining it in such a way that the point $\left(v_{1}, \ldots, v_{n}\right) \in \mathbb{R}^{n}$ is contained in the chamber $C_{x}, x=\left(v_{1}, \cdots . v_{n}\right)$. This is just one of the many ways in which our framework can be adapted in order to include different information.

Social decision rules Consider a population of $v$ agents. Each agent $i$ is characterized by a system of transitive preferences $\succeq_{i}$, i.e., transitive relations, over the set of social outcomes $X$. The set of systems of transitive preferences $\succeq_{i}$ is denoted by $\mathcal{P}$. Let $\mathcal{P}^{v}$ be the Cartesian product of $v$ copies of $\mathcal{P}$, a social decision rule $\mathcal{R}$ is a function

$$
\begin{aligned}
& \mathcal{R}: \mathcal{P}^{v}=\prod_{i=1}^{v} \mathcal{P} \longrightarrow \overline{\mathcal{P}} \\
&\left(\succeq_{1}, \ldots, \succeq_{v}\right) \longmapsto \succeq_{\mathcal{R}}\left(\succeq_{1}, \ldots, \succeq_{v}\right)
\end{aligned}
$$

which determines a system of social preferences $\succeq_{\mathcal{R}}\left(\succeq_{1}, \ldots, \succeq_{v}\right)$ from the preferences of $v$ individual agents. With $\overline{\mathcal{P}}$ we denote the set of systems of (not necessarily transitive $^{2}$ ) social preferences, i.e., a set of relations. Any type of social decision rule may fit into our framework, provided individual preferences are expressed sincerely. For the time being we rule out the possibility of strategic misrepresentation of a person's preferences.

In order to completely describe $\overline{\mathcal{P}}$, let us recall that if $\Delta=\{(x, x) \in X \times X\}$ is the diagonal of the Cartesian product $X \times X$, then an element $\succeq_{\mathcal{R}} \in \overline{\mathcal{P}}$ defines a subset

$$
Y_{1, \succeq_{\mathcal{R}}} \subset X \times X \backslash \Delta
$$

as follows: a pair $(x, y)$ is in $Y_{1, \succeq_{\mathcal{R}}}$ if and only if $x \succeq_{\mathcal{R}} y$.

Without loss of generality we will indicate by $\succeq_{\mathcal{R}} \in \overline{\mathcal{P}}$ a generic element in the image of $\mathcal{R}$.

We call $Y_{0, \succeq \mathcal{R}}$ the set of relevant social outcomes, i.e., the set of all social outcomes on which social preferences are expressed:

$$
Y_{0, \succeq_{\mathcal{R}}}=\left\{x \in X \mid \exists y:(x, y) \in Y_{1, \succeq \mathcal{R}} \text { or }(y, x) \in Y_{1, \succeq_{\mathcal{R}}}\right\} .
$$

${ }^{2}$ It is well known that individual transitivity does not guarantee transitivity for a social rule [6]. 
The subset $Y_{0, \succeq_{\mathcal{R}}} \subseteq X$ is in one-to-one correspondence with a subset $\mathcal{C}_{\succeq_{\mathcal{R}}} \subset \mathcal{C}$ of the set of chambers in $\mathcal{A}_{n, m}$ :

$$
C_{x} \in \mathcal{C}_{\succeq_{\mathcal{R}}} \Leftrightarrow x \in Y_{0, \succeq_{\mathcal{R}}}
$$

Example 2.3. Let us consider the space of social outcomes $X$ as in Example 2.1.Let us assume that the aggregated preferences of the group of friends upon the 3 features give rise to the following rule $\succ_{\mathcal{R}}$ :

(0.0.0) preferred to all, except $(1.1 .0) \succ_{\mathcal{R}}(0.0 .0),(0.0 .1) \succ_{\mathcal{R}}(0.0 .0) ;$

$(0.1 .0) \prec_{\mathcal{R}}(0.1 .1),(0.1 .0) \prec_{\mathcal{R}}(1.1 .1),(0.1 .0) \prec_{\mathcal{R}}(1.0 .0)$,

$(0.1 .0) \succ_{\mathcal{R}}(1.0 .1),(0.1 .0) \succ_{\mathcal{R}}(1.1 .0),(0.1 .0) \prec_{\mathcal{R}}(0.0 .1)$;

$(0.1 .1) \succ_{\mathcal{R}}(1.1 .1),(0.1 .1) \succ_{\mathcal{R}}(1.0 .0),(0.1 .1) \succ_{\mathcal{R}}(1.0 .1)$,

$(0.1 .1) \succ_{\mathcal{R}}(1.1 .0),(0.1 .1) \prec_{\mathcal{R}}(0.0 .1)$;

$(1.1 .1) \succ_{\mathcal{R}}(1.0 .0),(1.1 .1) \succ_{\mathcal{R}}(1.0 .1),(1.1 .1) \succ_{\mathcal{R}}(1.1 .0),(1.1 .1) \succ_{\mathcal{R}}(0.0 .1)$;

$(1.0 .0) \succ_{\mathcal{R}}(1.0 .1),(1.0 .0) \succ_{\mathcal{R}}(1.1 .0),(1.0 .0) \prec_{\mathcal{R}}(0.0 .1)$;

$(1.0 .1) \succ_{\mathcal{R}}(1.1 .0),(1.0 .1) \prec_{\mathcal{R}}(0.0 .1)$;

(1.1.0) $\prec \mathcal{R}(0.0 .1)$.

For the sake of simplicity, when possible, we will use the notation $v_{1} v_{2} v_{3}$ instead of $\left(v_{1} \cdot v_{2} . v_{3}\right)$. Thus $Y_{0, \succeq_{\mathcal{R}}}=\left\{v_{1} v_{2} v_{3} \mid v_{j}=0\right.$ or 1$\}=X$. Now $Y_{1, \succeq_{\mathcal{R}}}$ is given by all pairs $\left(v_{1} v_{2} v_{3}, g_{1} g_{2} g_{3}\right)$ such that $v_{1} v_{2} v_{3} \succ_{\mathcal{R}} g_{1} g_{2} g_{3}$; i.e., for example, $(000,101),(011,000) \in Y_{1, \succeq_{\mathcal{R}}}$ while $(000,011) \notin Y_{1, \succeq_{\mathcal{R}}}$.

Let us remark that when individual preferences are strict, as in the example above, then they are total orders on the space of relevant social outcomes $Y_{0, \succ_{\mathcal{R}}}$ (i.e., they are antisymmetric, transitive relations). While the aggregated preferences give rise, in general, to non-transitive relations, i.e., the rules $\succ_{\mathcal{R}}$ are not, in general, orders.

If social preferences are complete over $X$, then $Y_{0, \succeq_{\mathcal{R}}}=X$. In the sequel we will consider only complete preferences, although our framework may easily accommodate the more general case where $Y_{0, \succeq_{\mathcal{R}}} \subseteq X$.

Graphs We can represent the sets $Y_{0, \succeq_{\mathcal{R}}}$ and $Y_{1, \succeq_{\mathcal{R}}}$ respectively as the set of vertices and edges of an oriented graph $\mathcal{Y}_{\succeq_{\mathcal{R}}}$. Two vertices $x$ and $y$ in $Y_{0, \succeq_{\mathcal{R}}}$ are connected by an edge if and only if $(x, y) \in Y_{1, \succeq_{\mathcal{R}}}$ or $(y, x) \in Y_{1, \succeq_{\mathcal{R}}}$, while the orientation is from $x$ to $y$ in the former case and from $y$ to $x$ in the latter. In a natural way this construction applies to all subsets $Y \subset X \times X \backslash \Delta$.

Without loss of generality we will denote by $x$ the vertices of $\mathcal{Y}_{\succeq_{\mathcal{R}}}$ and by $(x, y)$ its edges.

Example 2.4. The social preferences of Example 2.3 are fully described by the oriented graph in Figure 2.1. 


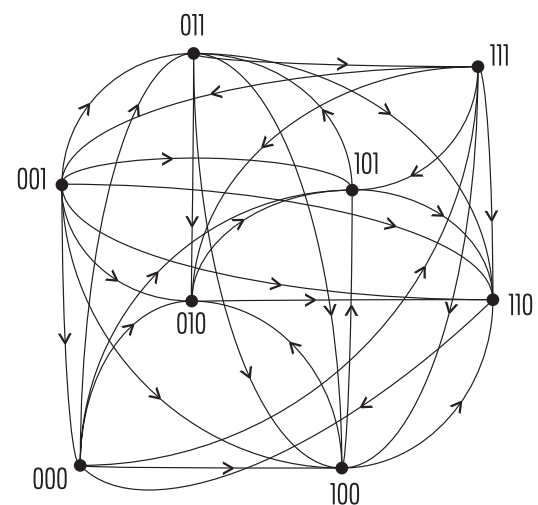

Figure 2.1. The oriented graph derived from Example 2.3.

Notice that the assumption of complete preferences guarantees that we will deal only with connected graphs.

A cycle of length $h$ in the oriented graph $\mathcal{Y}_{\succeq_{\mathcal{R}}}$ is a subgraph $\gamma_{I}$ with vertices $\left\{x_{1}, \ldots, x_{h}\right\}$ and edges $\left\{\left(x_{1}, x_{2}\right),\left(x_{2}, x_{3}\right), \ldots,\left(x_{h}, x_{1}\right)\right\}$. It corresponds to a Condorcet-Arrow cycle, i.e., to the sequence $x_{1} \succeq_{\mathcal{R}} \quad x_{2} \succeq_{\mathcal{R}} \ldots \succeq_{\mathcal{R}} x_{h} \succeq_{\mathcal{R}} x_{1}$. In Example 2.3 and in the corresponding graph of figure 2.1 we find a three outcomes cycle $000 \succ_{\mathcal{R}} 101 \succ_{\mathcal{R}} 011 \succ_{\mathcal{R}} 000$.

The Salvetti complex As shown in [23], we can put together the hyperplane arrangements theory and the graph description in a more general context that allows us to use both geometric and algebraic tools. Indeed we can construct a CWcomplex $\mathcal{S}(\mathcal{A})^{3}$ having the homotopy type of the complement $M(\mathcal{A})$ when the arrangement $\mathcal{A}$ is the complexification of a real one. In this complex each element $\succeq_{\mathcal{R}} \in \overline{\mathcal{P}}$ is equivalent to a subcomplex of the 1 -skeleton of $\mathcal{S}\left(\mathcal{A}_{\backslash, \mathbb{1}}\right)$.

Let us characterize the 0 and 1 -dimensional cells (also called 0 and 1 -skeletons, or vertices and edges) of the Salvetti complex and show how they are related to the graph $\mathcal{Y}_{\succeq_{\mathcal{R}}}$ for a given social rule $\succeq_{\mathcal{R}}$.

Let $\mathcal{A}_{n, m}$ be the arrangement associated to the space of social outcomes and $\mathcal{S}\left(\mathcal{A}_{n, m}\right)$ the correspondent Salvetti complex. The set of generators $\mathcal{S}_{0}\left(\mathcal{A}_{n, m}\right)$ of the 0 -skeleton of the complex $\mathcal{S}\left(\mathcal{A}_{n, m}\right)$ is in a one-to-one correspondence with the set of chambers in $\mathcal{A}_{n, m}$, i.e., with the set of social outcomes $X$. Thus

$$
\mathcal{S}_{0}\left(\mathcal{A}_{n, m}\right)=Y_{0, \succeq \mathcal{R}}=X
$$

for any given social rule $\succeq_{\mathcal{R}}$.

Example 2.5. The 0-skeleton $\mathcal{S}_{0}\left(\mathcal{A}_{3,2}\right)$ associated to Example 2.1 is given by 8 points that can be chosen freely one in each chamber of the coordinate arrangement $\mathcal{A}_{3,2}$, i.e., one in each octant. Thus they can be represented as the vertices of a cube or, more generally, as the vertices of a 3-dimensional polyhedron.

${ }^{3}$ In the Appendix we briefly recall the construction of the first boundary map of this complex. 
As remarked before, the vertex $x \in \mathbb{R}^{3}$ corresponding to the social outcome $x=v_{1} v_{2} v_{3}$ is not the point $\left(v_{1}, v_{2}, v_{3}\right), e . g ., 000$ is not the point $(0,0,0)$ which is the origin of the orthogonal system and hence does not lie in any octant.

Let us recall that two chambers $C_{x}$ and $C_{y}$ are said to be adjacent if and only if they are separated by only one hyperplane $H$. If $x$ and $y$ are the 0 -cells corresponding to $C_{x}$ and $C_{y}$, then we can consider the edge $(x, y)$ between them.

The generators of the 1-skeleton can be described as the elements in the set

$$
\mathcal{S}_{1}\left(\mathcal{A}_{n, m}\right)=\{(x, y) \in X \times X \backslash \Delta \mid x \text { and } y \text { are adjacent }\} .
$$

Two elements in $\mathcal{S}_{1}\left(\mathcal{A}_{n, m}\right)$ are consecutive if and only if the second entry of the first pair is equal to the first entry of the second pair: for example, $(x, y)$ and $(y, z)$. Given a subset of consecutive elements in $\mathcal{S}_{1}\left(\mathcal{A}_{n, m}\right)$

$$
\left\{\left(x_{1}, x_{2}\right),\left(x_{2}, x_{3}\right), \ldots,\left(x_{k-2}, x_{k-1}\right),\left(x_{k-1}, x_{k}\right)\right\} \in \mathcal{S}_{1}\left(\mathcal{A}_{n, m}\right)
$$

we define their formal sum as

$$
\left(x_{1}, x_{k}\right)=\sum_{j=1}^{k-1}\left(x_{j}, x_{j+1}\right) \text {. }
$$

Let $C_{x}$ and $C_{y}$ be two adjacent chambers separated by the hyperplane $H$. We say that we move from $C_{x}$ to $C_{y}$ across $H$ if we move along the edge $(x, y)$. Thus, by moving across hyperplanes, we can reach any chamber of the arrangement starting from a given one. If we only cross each hyperplane once, we call this path minimal. Obviously, there are many minimal paths, depending on which order we cross hyperplanes; however, they are all homotopically equivalent to each other [23].

It follows that, given a rule $\succeq_{\mathcal{R}}$, any edge $(x, y) \in Y_{1, \succeq_{\mathcal{R}}}$ can be written as a formal sum of a minimal number of consecutive elements in $\mathcal{S}_{1}\left(\mathcal{A}_{n, m}\right)$. The number of elements is exactly the number of hyperplanes that separate the two social outcomes $x, y \in X$.

Remark 2.6. Notice that the Salvetti complex is a CW-complex in $\mathbb{C}^{n}$, but it has an underlying real structure which is a purely simplicial complex. This structure can be used in order to recast and generalize some existing geometric models of voting such as those provided in [20].

\section{Object construction and social outcomes}

In this section we develop our main results. We define objects as bundles of features, and we show that, in general, by appropriate manipulations of the set of objects, almost every social outcome may be obtained from a given social rule (e.g., majority voting). Such an "object construction power", that is, the power of determining social outcomes by appropriately bundling or unbundling features, is stronger than 
the agenda power traditionally studied in the literature [16]. We then show that this power of manipulation also includes the possibility of breaking or creating intransitive cycles $\grave{a}$ la Condorcet-Arrow and of overturning the median voter effect. Finally we discuss the emerging trade-off between non manipulability and decidability. Coarser objects make social decisions less manipulable but they also increase the likelihood of intransitive cycles and the time required to reach a socially optimal outcome (if any). On the other hand finer objects make cycles less likely and allow reaching a social outcome more quickly, but the number of locally optimal social outcomes greatly increases and, therefore, the scope for manipulability becomes broader.

\subsection{Objects in social choice}

In this paragraph we give the main definitions and notation useful in the rest of the paper.

Objects schemes With the notation introduced above, let $\mathcal{A}_{n, m}$ be the hyperplane arrangement defined by $n$ features $F=\left\{f_{1}, \ldots, f_{n}\right\}$ indexed by the set $\{1, \ldots, n\}$ and $m$ possibilities for each of them. Let $\succeq_{\mathcal{R}}$ be the social preferences over the set of social outcomes $X$. Given a subset $I \subset\{1, \ldots, n\}$, an object $\mathcal{A}_{I}$ is a non empty subset of the arrangement $\mathcal{A}_{n, m}$ of the form

$$
\mathcal{A}_{I}=\left\{H_{i, j}\right\}_{\substack{0 \leq j<m-1 \\ 0 \in j<I}} .
$$

The cardinality of $\mathcal{A}_{I}$ is called the size of the object $\mathcal{A}_{I}$. We will also denote by $\mathcal{A}_{I^{c}}=\mathcal{A}_{n, m} \backslash \mathcal{A}_{I}$ the complement of the arrangement $\mathcal{A}_{I}$ in $\mathcal{A}_{n, m}$.

A set of objects $A=\left\{\mathcal{A}_{I_{1}}, \ldots, \mathcal{A}_{I_{k}}\right\}$ such that $\cup_{j=1}^{k} I_{j}=\{1, \ldots, n\}$ is an objects scheme. Notice that the subsets $I_{1}, \ldots, I_{k}$ do not have to partition the set $I$ as some index $h$ may belong to more than one subset. Since each subset $I_{j}$ naturally corresponds to a subset $\left\{f_{i}\right\}_{i \in I_{j}}$ of features, this is equivalent to notice that an objects scheme does not have to partition the set of features, as some of them may belong to more than one object. However, we require that the union of all objects covers all the features, otherwise the remaining features would not be decided on and could be simply omitted from the model.

Let $y$ be a social outcome in $X$, i.e., a chamber of $\mathcal{A}_{n, m}$, then the object instantiation $y\left(\mathcal{A}_{I}\right)$ is the chamber of the subarrangement $\mathcal{A}_{I}$ which contains the chamber corresponding to $y$, as shown, for instance, in Figure 3.1.

Moreover, the size of an objects scheme is the size of its largest object:

$$
|A|=\max \left\{\left|\mathcal{A}_{I_{1}}\right|, \ldots,\left|\mathcal{A}_{I_{k}}\right|\right\} .
$$

Neighbors of a social outcome Given an objects scheme $A=\left\{\mathcal{A}_{I_{1}}, \ldots, \mathcal{A}_{I_{k}}\right\}$, we say that a social outcome $y$ is a preferred neighbor of a social outcome $x$ with respect to an object $\mathcal{A}_{I_{h}} \in A$ if the following conditions hold:

1. $y \succeq_{\mathcal{R}} x$;

2. $y\left(\mathcal{A}_{I_{h}^{c}}\right)=x\left(\mathcal{A}_{I_{h}^{c}}\right)$, i.e., $x$ and $y$ belong to the same chamber of the arrangement $\mathcal{A}_{I_{h}^{c}}$ 

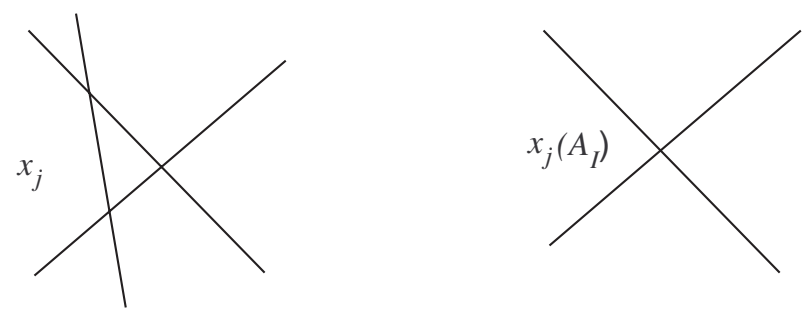

Figure 3.1. A graphic characterization of object instantiation.

3. $y\left(\mathcal{A}_{I_{h}}\right) \neq x\left(\mathcal{A}_{I_{h}}\right)$, i.e., $x$ and $y$ belong to different chambers of the arrangement $\mathcal{A}_{I_{h}}$.

In words, a preferred neighbor of a social outcome $x$ with respect to an object is another social outcome $y$ which is preferred to $x$ and differs from $x$ only in features that belong to that object. The set of all preferred neighbors of the social outcome $x$ with respect to $\mathcal{A}_{I_{h}} \in A$ is denoted by $\Phi\left(x, \mathcal{A}_{I_{h}}\right)$. The set of all preferred neighbors of the social outcome $x$ is denoted by $\Phi(x, A)=\bigcup_{j=1}^{k} \Phi\left(x, \mathcal{A}_{I_{j}}\right)$.

A social outcome $y \in \Phi\left(x, \mathcal{A}_{I_{h}}\right)$ is said to be a best neighbor of a social outcome $x$ with respect to an object $\mathcal{A}_{I_{h}} \in A$ if

$$
y \succeq_{\mathcal{R}} w \quad \forall w \in \Phi\left(x, \mathcal{A}_{I_{h}}\right) .
$$

The set of all best neighbors of the social outcome $x$ with respect to $\mathcal{A}_{I_{h}} \in A$ is denoted by $B\left(x, \mathcal{A}_{I_{h}}\right)$. The set of all best neighbors of the social outcome $x$ is denoted by $B(x, A)=\bigcup_{j=1}^{k} B\left(x, \mathcal{A}_{I_{j}}\right)$.

Paths and reachable social outcomes A path $P(x, y, A)$ through $A$, starting from $x$ and ending in $y$, is a sequence of best neighbors with respect to objects in $A$, i.e., a sequence

$$
x=x_{0} \preceq_{\mathcal{R}} x_{1} \preceq_{\mathcal{R}} \ldots \preceq_{\mathcal{R}} x_{s+1}=y
$$

such that there exist objects $\mathcal{A}_{I_{h_{1}}}, \ldots, \mathcal{A}_{I_{h_{s}}} \in A$ with $x_{i} \in B\left(x_{i-1}, \mathcal{A}_{I_{h_{i}}}\right)$ for all $1 \leqslant i \leqslant s$.

A social outcome $y$ is said to be reachable from $x$ with respect to an objects scheme $A$ if there exists a path $P(x, y, A)$.

Local optima A social outcome $x$ is said to be a local optimum for $A$ if and only if for all $y \in \Phi(x, A)$, one has $(x, y) \in Y_{1, \succeq_{\mathcal{R}}}$. In the case of strict preferences, this amounts to the condition $\Phi(x, A)=\emptyset$.

Agenda Let $A=\left\{\mathcal{A}_{I_{1}}, \ldots, \mathcal{A}_{I_{k}}\right\}$ be an objects scheme. An agenda $\alpha$ of $A$ is an ordered tuple of indices $\left(h_{1}, \ldots, h_{t}\right)$ such that the set $\left\{h_{1}, \ldots, h_{t}\right\}=\{1, \ldots, k\}$. An agenda $\alpha$ states the order in which objects $\mathcal{A}_{I_{i}}$ are decided upon. In general all objects of the given scheme appear at least once in an agenda, but they may appear more than once. In fact, because of non-separabilities, it is possible that 
after modifications of other objects, an object previously set into what appeared to be a locally optimal social outcome, may undergo further improvements. In order to study only the stable limit properties of agendas, we suppose that the agenda is repeated over and over again until either a local optimum or a cycle is reached.

The ordered tuple of objects $\left(\mathcal{A}_{I_{h_{1}}}, \ldots, \mathcal{A}_{I_{h_{t}}}\right)$ is denoted by $A_{\alpha}$. The set of all possible agendas of $A$ is denoted by $\Lambda(A)$. Let $\alpha=\left(h_{1}, \ldots, h_{t}\right)$ be an agenda. A path

$$
x_{0} \preceq_{\mathcal{R}} x_{1} \preceq \mathcal{R} \ldots \preceq \mathcal{R} x_{s}
$$

is said to be ordered along $\alpha$ if

$$
x_{i} \in B\left(x_{i-1}, \mathcal{A}_{I_{h_{q}+1}}\right)
$$

where $h_{q}$ is the remainder of the division of $i-1$ by $t$. Such a path will be denoted by $P\left(x_{0}, x_{s}, A_{\alpha}\right)$.

Maximal paths A path is said to be maximal if it ends in either a local optimum or a limit cycle, i.e., a cycle such that there is no social outcome not belonging to the cycle itself which is a preferred neighbor to any social outcome in the cycle. Thus a limit a cycle, once entered, cannot be exited.

Basin of attraction The basin of attraction $\Psi(x, A)$ of a local optimum $x$ with respect to an objects scheme $A$ is the set of the social outcomes $y$ such that there exists a maximal path $P(y, x, A)$ that ends up in $x$.

The ordered basin of attraction $\Psi\left(x, A_{\alpha}\right)$ of $x$ with respect to an agenda $\alpha$ of $A$ is the set of the social outcomes $y$ such that there exists a maximal path $P\left(y, x, A_{\alpha}\right)$ that ends up in $x$. Clearly, we have

$$
\Psi(x, A)=\bigcup_{\alpha \in \Lambda(A)} \Psi\left(x, A_{\alpha}\right) .
$$

Global optima A social outcome $z \in X$ is said to be a global optimum for an agenda $\alpha$ if $\Psi\left(z, A_{\alpha}\right)=X$ holds. It is said to be a global optimum for the objects scheme $A$ if and only if $\Psi\left(z, A_{\alpha}\right)=X$ holds for all agendas $\alpha \in \Lambda(A)$, i.e., it is a global optimum for all the agendas of $A$.

It is easy to verify that local and global optima strictly depend on the choice of the objects scheme $A$.

Example 3.1. Let us go back to Example 2.3 and denote by $H_{i}$ the hyperplane kernel of the polynomial $\alpha_{H_{i}}=\lambda_{i}$ for $i=1,2,3$. If the objects scheme is given by $A=\left\{\left\{H_{1}, H_{2}, H_{3}\right\}\right\}$, i.e., agents have to decide where, when and how simultaneously or by $A=\left\{\left\{H_{1}, H_{2}\right\},\left\{H_{3}\right\}\right\}$, i.e., agents decide where and when, given how, and, separately, they decide upon how, then the social rule always produces a cycle, that may be either $001 \succ_{\mathcal{R}} 011 \succ_{\mathcal{R}} 111 \succ_{\mathcal{R}} 001$ or $000 \succ_{\mathcal{R}} 010 \succ_{\mathcal{R}}$ $110 \succ_{\mathcal{R}} 000$ depending on the chosen agenda and initial condition. While if $A=\left\{\left\{H_{1}, H_{3}\right\},\left\{H_{2}\right\}\right\}$ or $A=\left\{\left\{H_{2}, H_{3}\right\},\left\{H_{1}\right\}\right\}$ then there exists a unique global optimum 001 for all agendas. 
The dependence of the optimum on the objects scheme is very strong. Indeed, it is easy to provide examples in which two different social outcomes $z_{1}, z_{2} \in X$ are global optima for two different choice of objects schemes. More precisely, there exist two objects schemes $A_{1}$ and $A_{2}$ such that $\Psi\left(z_{i}, A_{i}\right)=X$ and $\Psi\left(x, A_{i}\right)=\varnothing$ for all social outcomes $x \neq z_{i}$ and $i=1,2$.

Example 3.2. Let us consider the space of social outcomes $X$ as in Example 2.1 with the following preferences $\succ_{\mathcal{R}}$ :

$(0.0 .0)$ preferred to all except $(0.1 .1) \succ_{\mathcal{R}}(0.0 .0)$

(0.1.1) preferred to all except $(1.0 .1) \succ_{\mathcal{R}}(0.1 .1)$

$(1.0 .1) \prec_{\mathcal{R}}(1.0 .0),(1.0 .1) \succ_{\mathcal{R}}(1.1 .1),(1.0 .1) \succ_{\mathcal{R}}(0.0 .1)$,

$(1.0 .1) \prec_{\mathcal{R}}(1.1 .0),(1.0 .1) \prec_{\mathcal{R}}(0.1 .0)$;

$(0.0 .1) \succ_{\mathcal{R}}(1.1 .1),(0,0,1) \prec_{\mathcal{R}}(0,1,0),(0.0 .1) \prec_{\mathcal{R}}(1.1 .0),(0.0 .1) \prec_{\mathcal{R}}(1.0 .0) ;$

$(1.1 .0) \succ_{\mathcal{R}}(1.1 .1),(1,1,0) \succ_{\mathcal{R}}(1,0,0),(1.1 .0) \prec_{\mathcal{R}}(0.1 .0) ;$

$(1.1 .1) \prec_{\mathcal{R}}(0.1 .0),(1.1 .1) \prec_{\mathcal{R}}(1.0 .0)$;

$(0.1 .0) \succ_{\mathcal{R}}(1.0 .0)$.

With the objects scheme $A_{1}=\left\{\left\{H_{1}, H_{2}\right\},\left\{H_{3}\right\}\right\}$ the social outcome (0.0.0) is the unique global optimum, while with the objects scheme $A_{2}=\left\{\left\{H_{2}, H_{3}\right\},\left\{H_{1}\right\}\right\}$ the unique global optimum is (0.1.1).

From now on $A$ will be an objects scheme for a given social rule $\succ_{\mathcal{R}}$ on a social outcome space $X$ that corresponds to the arrangement $\mathcal{A}_{n, m}$.

A fundamental question is whether and under which conditions, given a social outcome $z \in X$, it is possible to choose an objects scheme $A$ such that the basin of attraction $\Psi(z, A)$ is not empty, i.e., such that $z$ is a local optimum or a global one for some objects scheme and some agenda. The answers to these questions are given in the next two sections.

\subsection{Objects and manipulability: local optimality}

Suppose one of the agents has a preferred social outcome $z \in X$ and some form of power in the determination of the objects, e.g., because he or she is chairing a committee or can somehow influence the cognitive framing of the choice, for instance through persuasion [18]. An interesting question is whether there exists an objects scheme $A=\left\{\mathcal{A}_{I_{1}}, \ldots, \mathcal{A}_{I_{k}}\right\}$ such that $z$ is a local optimum for $A$, and which combination of initial conditions and agenda will make social choice converge to $z$. The conditions under which this is possible will be provided in Theorem 3.4 that we will prove after introducing notions of separation and distance in hyperplane arrangements.

Let $x$ and $y$ be two social outcomes. They are said to be separated by an hyperplane $H \in \mathcal{A}_{n, m}$ if $H$ separates the chambers $C_{x}$ and $C_{y}$. In this case, the notation $x|H| y$ will be used. Moreover, $x$ and $y$ are said to be prominently separated if there exist two hyperplanes $H_{i_{1}, j_{1}}, H_{i_{2}, j_{2}} \in \mathcal{A}_{n, m}$ with $i_{1} \neq i_{2}$ (i.e., non-parallel) 
such that $x\left|H_{i_{1}, j_{1}}\right| y$ and $x\left|H_{i_{2}, j_{2}}\right| y$ hold. We will say that $x=\left(v_{1} \cdots v_{n}\right)$ and $y=\left(g_{1} \ldots . g_{n}\right)$ are separated by the feature $f_{i}$ if $v_{i} \neq g_{i}$, i.e., the value of the feature $f_{i}$ of $y$ differs from that of $x$. The set of the features that separate $x$ and $y$ is denoted by $\overline{\mathcal{H}}_{x, y}$.

Distance of social outcomes The distance between $x$ and $y$ is the minimum number of hyperplanes that separate $x$ from $y$. The prominent distance $d_{p}(x, y)$ is the number of features that separate $x$ from $y$, i.e., $\# \overline{\mathcal{H}}_{x, y}$. Note that $d_{p}(x, y)$ equals the minimum number of hyperplanes that prominently separate $x$ from $y$.

Recall that, by definition, if $H_{i, j}$ belongs to the object $\mathcal{A}_{I}$ for some $\bar{j}$, then $H_{i, j}$ belongs to $\mathcal{A}_{I}$ for all $0 \leqslant j<m_{i}-1$. Therefore, the subarrangement

$$
\mathcal{H}_{x, y}=\left\{H_{i, j} \in \mathcal{A}_{n, m} \mid i \in \overline{\mathcal{H}}_{x, y}, 0 \leqslant j<m_{i}-1\right\}
$$

of $\mathcal{A}_{n, m}$ has been considered. Note that, if we have $d_{p}(x, y)=1$ and $d(x, y)>1$, all the hyperplanes in $\mathcal{H}_{x, y}$ are parallel.

Remark 3.3. The sets $\mathcal{H}_{x, y}$ and $\overline{\mathcal{H}}_{x, y}$ are strictly interconnected. For instance, we will use the fact that $\mathcal{H}_{x, y}$ is contained in $\mathcal{H}_{z, w}$ if and only if $\overline{\mathcal{H}}_{x, y}$ is contained in $\overline{\mathcal{H}}_{z, w}$.

From now on, for the sake of simplicity, we will consider only social rules with strict preferences. This restriction is almost always unnecessary, but it greatly simplifies the presentation. Therefore, from now on, $\succ_{\mathcal{R}}$ will always denote a social rule with complete strict preferences.

If $z$ is a social outcome such that all social outcome $x_{1}, \ldots, x_{k}$ with $x_{j} \succ_{\mathcal{R}}$ $z$ verify $d_{p}\left(x_{j}, z\right)>1$, then $x_{j}$ and $z$ are prominently separated at least by two hyperplanes.

It follows that we can build an objects scheme $A_{z}$ such that $\mathcal{H}_{x_{j}, z} \nsubseteq \mathcal{A}$ for all $\mathcal{A} \in A_{z}$ and all $1 \leq j \leq k$. For example, if $H_{j}^{1}, H_{j}^{2} \in \mathcal{H}_{x_{j}, z}$ are two hyperplanes related to different features for $1 \leq j \leq k$, then let us consider an objects scheme $A_{z}$ such that for any $x_{j}$ there exist two objects $\mathcal{A}_{j}^{1}, \mathcal{A}_{j}^{2}$ in $A_{z}$ with $H_{j}^{1} \in \mathcal{A}_{j}^{1}, H_{j}^{2} \in \mathcal{A}_{j}^{2}$ and $\left\{H_{j}^{1}, H_{j}^{2}\right\} \nsubseteq \mathcal{A}$ for all $\mathcal{A} \in A_{z}$.

It is obvious that such an objects scheme exists. Moreover $z$ is a local optimum for $A_{z}$. Indeed for all $x_{j} \succ_{\mathcal{R}} z$ and for all $\mathcal{A} \in A_{z}$ the chambers $C_{x_{j}}\left(\mathcal{A}^{c}\right)$ and $C_{z}\left(\mathcal{A}^{c}\right)$ are always separated by $H_{j}^{1}$ or $H_{j}^{2}$. That is $x_{j}\left(\mathcal{A}^{c}\right) \neq z\left(\mathcal{A}^{c}\right)$ and then $\Phi(z, \mathcal{A})=\emptyset$ for all $\mathcal{A} \in A_{z}$. It follows that $z \in \Psi\left(z, A_{z}\right)$ and then $z \in \Psi\left(z, A_{z, \alpha}\right)$ for all agendas $\alpha$, i.e., $z$ is a local optimum.

On the other hand if $x$ is a social outcome $x \succ_{\mathcal{R}} z$ such that $d_{p}(x, z)=1$ then for any objects scheme $A$ there is at least one object $\mathcal{A}$ such that all hyperplanes $H$ separating $x$ from $z$ are in $\mathcal{A}$. Then, by definition, $x \in \Phi(z, \mathcal{A}) \neq \emptyset$. Hence we get the following easy fact.

Theorem 3.4. Let $z$ be a social outcome. Then there exists an objects scheme $A_{z}$ for which $z$ is a local optimum if and only if the inequality $d_{p}(w, z)>1$ holds for any social outcome $w$ with $w \succ z$. 
Remark 3.5. Theorem 3.4 also tells us that the condition for a social outcome to be a local optimum does not depend on the agenda. If $z$ is a local optimum for an agenda $\alpha \in \Lambda(A)$ it will be a local optimum also for any other agenda $\beta \in \Lambda(A)$, Thus object construction power is, in some sense, stronger than agenda power. Notice, however, that the basin of attraction of a local optimum is, in general, different for different agendas and, therefore, even when starting from the same initial social outcome the choice processes may end up in different local optima for different agendas.

Moreover in order to verify if a social outcome is a local optimum we need only to verify if it is greater than all the $n \cdot m-1$ social outcomes with prominent distance equal one from it, instead of checking all $m^{n}-1$ social outcomes in $X$. The above construction implicitly gives also an algorithmic description of how to construct an objects scheme which makes social choice converge to a desired local optimum.

Finally, notice that the independence of our construction from the 1-dimensional distance, i.e., the distance along the 1-dimensional family of hyperplanes $\left\{H_{i, j}\right\}_{0 \leq j<m-1}$ for a fixed $i$, is a consequence of the independence of the choice from the order in which a 1-dimensional list of objects is given.

Example 3.6. Let $\succ_{\mathcal{R}}$ be the social decision rule of Example 3.2. Thanks to Theorem 3.4 it is very simple to verify that $(0.0 .0)$ and $(0.1 .1)$ are the only local optima for $\succ_{\mathcal{R}}$. Moreover, exploiting the above procedure, we can construct all objects schemes for which (0.0.0) and (0.1.1) are reachable. Hence we can notice that, if $A_{1}$ and $A_{2}$ are the objects schemes of Example 3.2, then their basins of attraction coincide with $X, \Psi\left((0.0 .0), A_{1}\right)=X$ and $\Psi\left((0.1 .1), A_{2}\right)=X$, i.e., $(0.0 .0)$ and (0.1.1) are global optima for, respectively, object schemes $A_{1}$ and $A_{2}$. On the contrary, if we consider for instance the objects scheme $A_{3}=\left\{\left\{H_{1}\right\},\left\{H_{2}\right\},\left\{H_{3}\right\}\right\}$ then $(0.0 .0)$ and (0.1.1) are both reachable local optima, each of them with its own basin of attraction such as $\Psi\left((0.0 .0), A_{3}\right) \subset X$ and $\Psi\left((0.1 .1), A_{3}\right) \subset X$.

If a social outcome $z$ does not meet the necessary and sufficient condition of Theorem 3.4 it cannot be a local optimum. However, an agent with object construction power may nevertheless make choices converge to another social outcome close enough to $z$.

Free social outcome We say that a social outcome $z$ is free with respect to a social decision rule $\succ_{\mathcal{R}}$ if and only if for any social outcome $x$ such that $x \succ_{\mathcal{R}} z$ then $d_{p}(x, z)>1$. Thus, by Theorem $3.4, z$ is the local optimum for an objects scheme $A_{z}$ if and only if $z$ is free.

Moreover a social outcome $\bar{z}$ has minimal distance from $\mathrm{z}$ with respect to $\succ_{\mathcal{R}}$ if and only if $\bar{z}$ is free with respect to a decision rule $\mathcal{R}$ and

$$
d(z, \bar{z})=\min \{d(z, x) \mid x \text { is free }\} .
$$

Thus it is always possible to build an objects scheme $A_{\bar{z}}$ such that the social outcome $\bar{z}$ of minimal distance from $\mathrm{z}$ with respect to $\succ_{\mathcal{R}}$ is a local optimum. 
Remark 3.7. If we consider the classical 1-dimensional problem, then the prominent distance between two social outcomes $x$ and $z$ is always $d_{p}(x, z)=1$. It follows that:

- The social outcome $z$ is free if and only if $z$ is an optimum, i.e., for any social outcome $x, z \succ_{\mathcal{R}} x$;

- If the social outcome of minimal distance from $\mathrm{z}$ with respect to $\succ_{\mathcal{R}}$ exists then it is the only optimum;

- If the social outcome of minimal distance from $\mathrm{z}$ with respect to $\succ_{\mathcal{R}}$ does not exist, then our theorem simply recovers the usual intransitive cycles.

\subsection{Objects and manipulability: global optimality}

We now analyze under which conditions a social outcome $z$ is a global optimum for an agenda $\alpha$ and under which conditions it is a global optimum for all agendas $\alpha \in \Lambda(A)$.

From now on, given a social outcome $z \in X$, we will denote by $A_{z}$ an objects scheme such that $\Phi\left(z, A_{z}\right)=\emptyset$, i.e., it admits $z$ as local optimum.

Let us remark that, given a social outcome $z \in X$, if $z$ is a global optimum for an agenda $\alpha \in \Lambda\left(A_{z}\right)$ then the following two conditions hold:

1. if $x \in X$ is a social outcome such that $x \succ_{\mathcal{R}} z$ then there exist social outcomes $\left\{x_{1}, \ldots, x_{k}\right\}$ such that

$$
z \prec \mathcal{R} x \prec_{\mathcal{R}} x_{1} \prec_{\mathcal{R}} \ldots \prec_{\mathcal{R}} x_{k} \prec \mathcal{R} z
$$

Indeed if this is not the case, all paths starting from $x$ would never end up in $z$ for all possible objects schemes $A$; i.e., $x \notin \Psi(z, A)$ for any objects scheme $A$; 2. $\Phi\left(x, A_{z}\right) \neq \emptyset$ for all social outcomes $x \neq z$. Otherwise if $\Phi\left(x, A_{z}\right)=\emptyset$, then $x \in \Psi\left(x, A_{z, \alpha}\right)$ for all agendas $\alpha \in \Lambda\left(A_{z}\right)$.

The first condition is simple to verify. The second one is true for all social outcomes $x \in X$ that are not free (see Theorem 3.4), while free social outcomes must satisfy the following conditions.

Theorem 3.8. Let $z$ be a free social outcome. Then there exists an objects scheme $A_{z}$ such that $\Phi\left(z, A_{z}\right)=\emptyset$ and $\Phi\left(x, A_{z}\right) \neq \emptyset$ for all free social outcomes $x$ if and only if the condition

$$
\exists y>x \text { such that } \mathcal{H}_{w, z} \nsubseteq \mathcal{H}_{x, y} \quad \forall w \succ z
$$

holds for all free social outcomes $x \neq z$. 
Proof. Sufficiency: If $\Phi\left(x, A_{z}\right) \neq \emptyset$ then there exists a social outcome $y$ in $\Phi\left(x, A_{z}\right)$. By construction $y$ satisfies the following two conditions:

1. $y \succ_{\mathcal{R}} x$

2. There exists $\mathcal{A} \in A_{z}$ such that $\mathcal{H}_{x, y} \subset \mathcal{A}$ or, equivalently, $\mathcal{H}_{x, y}^{c} \supset \mathcal{A}^{c}$.

Moreover $\Phi\left(z, A_{z}\right)=\emptyset$ implies that for all objects $\mathcal{A} \in A_{z}$ and for all $w \succ_{\mathcal{R}} z$ the intersection $\mathcal{A}^{c} \cap \mathcal{H}_{w, z} \neq \emptyset$, then $\mathcal{H}_{x, y}^{c} \cap \mathcal{H}_{w, z} \neq \emptyset$, i.e., $\mathcal{H}_{w, z} \nsubseteq \mathcal{H}_{x, y}$ for all $w \succ_{\mathcal{R}} z$. Hence $y$ is the social outcome verifying condition (3.3).

Necessity: Let $G\left(z, A_{z}\right) \subset X$ be the set

$$
G\left(z, A_{z}\right)=\left\{x \in X \mid \Phi\left(x, A_{z}\right)=\emptyset\right\}
$$

i.e., if $x \in G\left(z, A_{z}\right)$ then it is a local optimum for $A_{z}$. If $G\left(z, A_{z}\right)=\{z\}$ we proved necessity, otherwise given $x \in G\left(z, A_{z}\right)$ we can build a new objects scheme $A_{z}^{\prime}$ as follows. Let $y \succ_{\mathcal{R}} x$ as in the hypothesis, then we can consider the new objects scheme $A_{z}^{\prime}=A_{z} \cup \mathcal{H}_{x, y}$.

Clearly $y \in \Phi\left(x, \mathcal{H}_{x, y}\right)$, i.e., $x$ is not a local optimum for $A_{z}^{\prime}$ while $z$ is still a local optimum for $A_{z}^{\prime}$. Indeed $\Phi(z, \mathcal{A})=\emptyset$ for all $\mathcal{A} \in A_{z}$ by construction while $\Phi\left(z, \mathcal{H}_{x, y}\right)=\varnothing$ by hypothesis: let $w$ be a social outcome in $\Phi\left(z, \mathcal{H}_{x, y}\right) \neq \emptyset$. Then $w \succ_{\mathcal{R}} z$ and $\mathcal{H}_{w, z} \subset \mathcal{H}_{x, y}$, but this is an absurd since $\mathcal{H}_{w, z} \nsubseteq \mathcal{H}_{x, y}$ for all $w \succ_{\mathcal{R}} z$ by condition (3.3).

We have a new objects scheme $A_{z}^{\prime}$ such that $z$ is a local optimum for $A_{z}^{\prime}$ and $G\left(z, A_{z}^{\prime}\right) \subset G\left(z, A_{z}\right) \backslash\{x\}$. We can iterate this construction until we obtain an objects scheme $A$ such that $z$ is a local optimum and $G(z, A)=\{z\}$.

The above theorem gives us a necessary and sufficient condition for the existence of an objects scheme $A_{z}$ such that for all possible agendas and all possible starting social outcomes $x \in X$ the voting process ends up in $z$ or in a cycle. Following the proof of this theorem we can also provide [1] an algorithm which computes such an objects scheme $A_{z}$. Moreover, given a starting social outcome $x \in X$, condition (3.3) allows us to construct an objects scheme $A_{z}$ and an agenda $\alpha$ in $A$ such that $x \in \Psi\left(z, A_{z, \alpha}\right)$.

Example 3.9. Let us consider the social outcome $z=(0.0 .0)$ and the social rule $\succ_{\mathcal{R}}$ as in Example 3.2. Thanks to Theorem 3.8 it is easy to prove the existence of an objects scheme $A$ such that (0.0.0) is the only possible optimum. Indeed the only social outcome $x$ preferred to $z$, i.e., $x \succ_{\mathcal{R}} z$, is $x=(0.1 .1)$ and the social outcome $(1.0 .1)=y \succ_{\mathcal{R}} x$ verifies

$$
\mathcal{H}_{x, z}=\left\{H_{2}, H_{3}\right\} \nsubseteq \mathcal{H}_{x, y}=\left\{H_{1}, H_{2}\right\} .
$$

Then, by Theorem 3.8, there is an objects scheme $A_{z}$ for which $z$ is the only possible optimum. Hence or $z$ is a global optimum for the objects scheme $A_{z}$, i.e., $\Psi(z, A)=$ $X$, or there is a social outcome $x \in X$ such that any path through $A_{z}$ starting from $x$ ends up in a cycle.

The same argument applies for the social outcome $z=(0.1 .1)$. 
Example 3.10. Let us consider the space of social outcomes $X$ as in Example 2.1 with the following preferences $\succ_{\mathcal{R}}$ :

(0.0.0) preferred to all except $(0.1 .1) \succ_{\mathcal{R}}(0.0 .0)$

(0.1.1) preferred to all except (1.0.0) $\succ_{\mathcal{R}}(0.1 .1)$

$(1.0 .1) \prec_{\mathcal{R}}(1.0 .0),(1.0 .1) \succ_{\mathcal{R}}(1.1 .1),(1.0 .1) \succ_{\mathcal{R}}(0.0 .1)$,

$(1.0 .1) \prec_{\mathcal{R}}(1.1 .0),(1.0 .1) \prec_{\mathcal{R}}(0.1 .0)$

$(0.0 .1) \succ_{\mathcal{R}}(1.1 .1),(0.0 .1) \prec_{\mathcal{R}}(0.1 .0),(0.0 .1) \prec_{\mathcal{R}}(1.1 .0),(0.0 .1) \prec_{\mathcal{R}}(1.0 .0)$

$(1.1 .0) \succ_{\mathcal{R}}(1.1 .1),(1.1 .0) \succ_{\mathcal{R}}(1.0 .0),(1.1 .0) \prec_{\mathcal{R}}(0.1 .0)$

$(1.1 .1) \prec_{\mathcal{R}}(0.1 .0),(1.1 .1) \prec_{\mathcal{R}}(1.0 .0)$

$(0.1 .0) \succ_{\mathcal{R}}(1.0 .0)$.

The social outcome $z=(0.0 .0)$ is a local optimum by Theorem 3.4, but it is not a global optimum for any objects scheme. Indeed the social outcome $x=(0.1 .1)$ is a free outcome and it verifies $x \succ_{\mathcal{R}} z$. Moreover $y=(1.0 .0)$ is the only social outcome preferred to $x$, i.e., $y \succ_{\mathcal{R}} x$, and it verifies

$$
\mathcal{H}_{x, z}=\left\{H_{2}, H_{3}\right\} \subset \mathcal{H}_{x, y}=\left\{H_{1}, H_{2}, H_{3}\right\} .
$$

Then, by Theorem 3.8, it follows that if (0.0.0) is a local optimum for an objects scheme $A$, then also (0.1.1) is a local optimum for $A$ and hence $(0.1 .1) \notin$ $\Psi((0.0 .0), A)$.

\subsection{Objects and manipulability: obtaining a social outcome from a given status quo}

So far we have analyzed cases in which objects, agendas and the initial status quo may all be manipulated in order to obtain a desired social outcome. In this section instead we assume that the initial status quo is exogenously given and only objects and agenda are subject to manipulation. In Theorem 3.12 we give a necessary and sufficient condition that a status quo $x$ needs to satisfy in order to belong to the basin of attraction of a chosen social outcome $z$ with respect to some objects scheme $A$. In other words, given an agent with preferred free social outcome $z$, we find the conditions under which he or she may obtain $z$ from the given initial condition.

Universal basin of attraction Let $\Pi\left(\mathcal{A}_{n, m}\right)$ be the set of all possible objects schemes in $\mathcal{A}_{n, m}$. The universal basin of attraction of a social outcome $z \in X$ is the set

$$
\Psi(z)=\bigcup_{A \in \Pi\left(\mathcal{A}_{n, m}\right)} \Psi(z, A),
$$

i.e., the set of all the social outcomes $x$ such that there exists an objects scheme for which there is a path starting from $x$ and ending up in $z$.

By Theorem 3.4, the universal basin of attraction of the social outcome $z$ is non-empty if and only if $z$ is free. 
As above, let $x \in X$ be a starting social outcome. Let us define the following set:

$$
G_{x}^{z}=\left\{y \succ_{\mathcal{R}} x \mid \mathcal{H}_{w, z} \nsubseteq \mathcal{H}_{x, y} \quad \forall w \succ_{\mathcal{R}} z \text { and } B\left(x, \mathcal{H}_{x, y}\right) \neq \emptyset\right\}
$$

Proposition 3.11. Given two social outcomes $x, z \in X$, if $x$ is in the universal basin of attraction $\Psi(z)$ then $G_{x}^{z} \neq \emptyset$.

Proof. Let us assume that $G_{x}^{z}$ is empty. Then either:

i) condition 3.3 is not met by any of the social outcomes $y \in X$. Therefore, by Theorem 3.8, it follows that $\Phi\left(x, A_{z}\right)=\emptyset$ for all $A_{z}$ such that $\Phi\left(z, A_{z}\right)=\emptyset$, i.e. if $x$ is the starting social outcome then all maximal paths in $A_{z}$ end up in $x$ for any agenda $\alpha$; or:

ii) $\Phi\left(x, A_{z}\right) \neq \emptyset$ but $B\left(x, A_{z}\right)=\emptyset$ for all objects schemes $A_{z}$ such that $\Phi\left(z, A_{z}\right)=$ $\emptyset$, i.e., if $x$ is the starting social outcome then all voting processes end up in a cycle.

In both cases $x \notin \Psi(z)$ and this concludes the proof.

Suppose now that $x$ is a social outcome such that $G_{x}^{z}$ is non-empty (therefore $z$ is free $\left.{ }^{4}\right)$. If $B\left(x, \mathcal{H}_{x, y}\right)$ is non-empty, its cardinality is one. The only element of $B\left(x, \mathcal{H}_{x, y}\right)$ will be denoted by $b_{x, y}$, and we can define the set

$$
B G_{x}^{z}=\left\{b_{x, y} \mid y \in G_{x}^{z}\right\}
$$

Then we can consider the following finite subsets ${ }^{5}$ of $X$

$$
\begin{aligned}
& E_{0}^{z}=\{z\} \\
& E_{1}^{z}=\left\{x \in X \backslash\{z\} \mid z \in B G_{x}^{z}\right\} \\
& E_{2}^{z}=\left\{x \in X \backslash \cup_{i=0}^{1} E_{i}^{z} \mid E_{1}^{z} \cap B G_{x}^{z} \neq \emptyset\right\} \\
& \vdots \\
& E_{h}^{z}=\left\{x \in X \backslash \cup_{i=0}^{h-1} E_{i}^{z} \mid E_{h-1}^{z} \cap B G_{x}^{z} \neq \emptyset\right\} \\
& E_{h+1}^{z}=\left\{x \in X \backslash \cup_{i=0}^{h} E_{i}^{z} \mid E_{h}^{z} \cap B G_{x}^{z} \neq \emptyset\right\}=\emptyset
\end{aligned}
$$

where $h$ is the smallest integer such that $E_{h+1}^{z}$ is empty, and

$$
E^{z}=\bigcup_{i=1}^{h} E_{i}^{z}
$$

${ }^{4}$ For the sake of completeness, we assume $G_{x}^{z}$ to be $\emptyset$ if $z$ is not free.

5 Once again, for the sake of completeness, we assume all these sets to be empty if $z$ is not free. 
We have the following:

Theorem 3.12. Let $x$ and $z$ be two social outcomes. Then $x$ is in the universal basin of attraction $\Psi(z)$ if and only if $x$ belongs to $E^{z}$, i.e.

$$
\Psi(z)=E^{z} .
$$

Proof. Sufficiency: The proof is by induction. If $x \in \Psi(z)$, then there exists an objects scheme $A=\left\{\mathcal{A}_{I_{1}}, \ldots, \mathcal{A}_{I_{t}}\right\}$ such that $x \in \Psi(z, A)$ and an agenda $\alpha=$ $\left(h_{k}, \ldots, h_{1}\right)$ for $A$ such that the ordered path along $\alpha$

$$
x=x_{k} \prec \mathcal{R} x_{k-1} \prec \mathcal{R} \ldots \prec_{\mathcal{R}} x_{1} \prec \mathcal{R} x_{0}=z
$$

is maximal.

The social outcome $x_{0}=z$ is in $E^{z}$ and, by induction, we assume that there is a $0 \leq j \leq k$ such that $x_{j} \in E^{z}$, i.e., $x_{j} \in E_{i}^{z}$ for an integer $0 \leq i \leq h$. By construction $x_{j} \in B\left(x_{j+1}, \mathcal{A}_{h_{j+1}}\right)$ then $x_{j} \in B\left(x_{j+1}, \mathcal{H}_{x_{j+1}, x_{j}}\right) \neq \emptyset$. Moreover $\mathcal{H}_{w, z} \nsubseteq \mathcal{H}_{x_{j+1}, x_{j}} \quad \forall w \succ_{\mathcal{R}} z$, indeed if $w \succ_{\mathcal{R}} z$ is a social outcome such that $\mathcal{H}_{w, z} \subseteq \mathcal{H}_{x_{j+1}, x_{j}} \subseteq \mathcal{A}_{h_{j}}$ then $z \notin \Psi(z, A)$ which is absurd. It follows that $x_{j} \in$ $B G_{x_{j+1}}^{z}$.

Since $x_{j} \in E_{i}^{z} \cap B G_{x_{j+1}}^{z} \neq \emptyset$ then either $x_{j+1} \in E_{i+1}^{z}$ or $x_{j+1} \in \cup_{s=0}^{i-1} E_{s}^{z}$, i.e., $x_{j+1} \in E^{z}$.

Necessity: Viceversa, if $x \in E^{z}$ then there is an integer $0 \leq i \leq h$ such that $x \in E_{i}^{z}$ and we can construct a path

$$
x=x_{i} \prec \mathcal{R} x_{i-1} \prec \mathcal{R} \ldots \prec \mathcal{R} x_{1} \prec \mathcal{R} x_{0}=z
$$

such that $x_{j} \in E_{j}^{z} \cap B G_{x_{j+1}}$ for $0 \leq j \leq i-1$.

Let us consider an objects scheme $A_{z}$ such that $z \in \Psi\left(z, A_{z}\right)$, then

$$
A=\left\{\mathcal{H}_{x_{j}, x_{j+1}}\right\}_{0 \leq j \leq i-1} \cup A_{z}
$$

still satisfies $z \in \Psi(z, A)$. Moreover let $\alpha=\left(h_{1}, \ldots, h_{k}\right)$ be an agenda for $A$ such that the $h_{t}$-object in the ordered scheme $A_{\alpha}$ is $\mathcal{H}_{x_{i-t-1}, x_{i-t}}$ for $0 \leq t \leq i-1$. Then, by construction, the path (3.6) is exactly the maximal path $P\left(x, z, A_{\alpha}\right)$ and $x \in \Psi(z)$.

Example 3.13. Let us consider again the social outcome $z=(0.0 .0)$ and the social decision rule $\succ_{\mathcal{R}}$ as in Example 3.2. The social outcome $w=(0.1 .1)$ is the only outcome preferred to $z$ and $\mathcal{H}_{w, z}=\left\{H_{2}, H_{3}\right\}$. It is easy to verify that:

$$
\begin{aligned}
& E_{0}^{z}=\{(0.0 .0)\} \\
& E_{1}^{z}=X \backslash\{(0.0 .0),(0.1 .1),(1.1 .1)\} \\
& E_{2}^{z}=\{(0.1 .1),(1.1 .1)\} .
\end{aligned}
$$

Then the universal basin of attraction $\Psi(z)$ is the whole set $X$.

With the above notation, if we consider the social rule $\succ_{\mathcal{R}}$ defined in Example 3.10 , then $G_{w}^{z}=\emptyset$, hence $w=(0.1 .1) \notin E^{z}$. 


\subsection{Breaking intransitive cycles}

In this section we show that also intransitive cycles which, as well known, can be generated by any social aggregation rule are subject to manipulation through object construction. The main result of this section, Theorem 3.14, demonstrates that almost any intransitive cycle can be broken by appropriate modification of the objects scheme. It actually turns out that cycles can be broken by introducing new local optima. Thus, we observe a trade-off between decidability, i.e., the possibility of reaching some social optimum in a feasible time, and non-manipulability, that is, the convergence of the social decision process to a unique global outcome that does not depend on initial condition and agenda. The balance in this trade-off is struck by the objects scheme. In particular, we will show that coarse objects containing many features tend to produce many cycles and few local optima, whereas fine objects containing only one or a few features are much less likely to induce cycles but tend to generate many local optima and, therefore, greatly increase the opportunity for manipulation.

Suppose that for some objects scheme $A^{\prime}$ social preferences may encounter a set of cycles $\Gamma$. We will say that an objects scheme $A \neq A^{\prime}$ breaks the cycles $\Gamma$ if social choice does not enter into any cycle belonging to $\Gamma$ with the new objects scheme.

Given a cycle $\gamma: x_{1} \prec_{\mathcal{R}} \ldots \prec_{\mathcal{R}} x_{k} \prec_{\mathcal{R}} x_{k+1}=x_{1}$, let us define the following subset of hyperplanes in $\mathcal{A}_{n, m}$ :

$$
\mathcal{H}_{\gamma}=\bigcup_{1 \leq j \leq k+1} \mathcal{H}_{x_{j}, x_{j+1}} .
$$

We will say that a cycle $\gamma: x_{1} \prec_{\mathcal{R}} \ldots \prec_{\mathcal{R}} x_{k} \prec_{\mathcal{R}} x_{k+1}=x_{1}$ is free if there are at least two consecutive indices $j, j+1$ such that $d_{p}\left(x_{j}, x_{j+1}\right)>1$.

Then we have the following:

Theorem 3.14. Let $\mathcal{Y}_{\succ_{\mathcal{R}}}$ be the oriented graph for a given social rule $\succ_{\mathcal{R}}$ and $\mathcal{A}_{n, m}$ the arrangement associated to the space of social outcomes. Then for any given set $\Gamma=\left\{\gamma_{1}, \ldots, \gamma_{h}\right\}$ of free cycles in $\mathcal{Y}_{\succ_{\mathcal{R}}}$ there exists an objects scheme A such that all voting process will never end up in any cycle of $\Gamma$. Moreover this scheme can be chosen with maximum size.

In order to prove this theorem we remark that if $\gamma \in \mathcal{Y}_{\succ_{\mathcal{R}}}$ is a cycle and $A$ an objects scheme such that

1. $\mathcal{H}_{\gamma} \nsubseteq \mathcal{A}$ for any object $\mathcal{A} \in A$,

2. any path ordered along $\alpha$ and starting in $x$ does not end up in $\gamma$ for any agenda $\alpha$,

then $\gamma$ disappears in $A$.

Lemma 3.15. Let $\gamma \in \mathcal{Y}_{\succ_{\mathcal{R}}}$ be a cycle and $A$ an objects scheme such that at least two consecutive outcomes $x_{j} \prec_{\mathcal{R}} x_{j+1}$ in the cycle satisfy $\mathcal{H}_{x_{j}, x_{j+1}} \nsubseteq \mathcal{A}$ for any object $\mathcal{A} \in A$. Then $\gamma$ disappears in $A$. 
Proof. Condition 1 above is trivially satisfied. Moreover, by hypothesis, let $x_{j} \prec \mathcal{R}$ $x_{j+1}$ be two consecutive outcomes in $\gamma$ such that $\mathcal{H}_{x_{j}, x_{j+1}} \nsubseteq \mathcal{A}$ for any object $\mathcal{A} \in A$. Then, by definition, $x_{j+1} \notin B\left(x_{j}, \mathcal{A}\right)$ for all objects $\mathcal{A} \in A$, i.e., it is not possible to move from $x_{j}$ to $x_{j+1}$ directly and then condition 2 holds. This concludes the proof.

As a direct consequence of the above lemma any free cycle $\gamma \in \mathcal{Y}_{\succ_{\mathcal{R}}}$ disappears for the trivial object scheme

$$
A=\left\{\left\{H_{i, j}\right\} \underset{\substack{1 \leq i \leq n \\ 0 \leq j<m-1}}{ }\right\} .
$$

Proof of Theorem 3.14. Let us consider the finite set $\mathbb{A}_{\Gamma}$ of all possible objects schemes $A$ of $\mathcal{A}_{n, m}$ such that all voting processes in $A$ will never end up in any cycle in $\Gamma$. This set is not empty, indeed the trivial objects scheme

$$
A=\left\{\left\{H_{i, j}\right\} \underset{\substack{1 \leq i \leq n \\ 0 \leq j<m-1}}{0 \leq m}\right\}
$$

is such that any social choice process will never end up in any cycle in $\Gamma$. This concludes the proof. Moreover the set of the sizes of elements $A \in \mathbb{A}_{\Gamma}$ is a finite non-empty set of natural numbers $\mathbb{N}$ and then it admits a maximum.

Example 3.16. In Example 3.1 the objects schemes $A=\left\{\left\{H_{1}, H_{3}\right\},\left\{H_{2}\right\}\right\}$ and $A=\left\{\left\{H_{2}, H_{3}\right\},\left\{H_{1}\right\}\right\}$ break at once all cycles in the graph $\mathcal{Y}_{\succ_{\mathcal{R}}}$ of Example 2.3.

Remark 3.17. A cycle $\gamma$ which is not free can also be broken in most cases, but not always. An example is given by the cycle $\gamma: 00 \prec_{\mathcal{R}} 01 \prec_{\mathcal{R}} 11 \prec_{\mathcal{R}} 10 \prec_{\mathcal{R}}$ 00 in the case $\mathcal{A}_{2,1}$. Indeed, in this case, the only two objects schemes are $A=$ $\left\{\left\{H_{1}, H_{2}\right\}\right\}$ and $A=\left\{\left\{H_{1}\right\},\left\{H_{2}\right\}\right\}$ and none of them breaks $\gamma$.

The above theorem proves the existence of a non-trivial objects scheme $A$ such that all the cycles in a given set $\Gamma=\left\{\gamma_{1}, \ldots, \gamma_{h}\right\}$ are broken, while Lemma 3.15 shows how to construct it.

All in all, a cycle $\gamma$ may be broken, i.e., it disappears for a given objects scheme $A$, if there exist consecutive social outcomes $x, w$ involved in the cycle $\gamma$ such that $\mathcal{H}_{x, w} \nsubseteq \mathcal{A}$ for all $\mathcal{A}$ belonging to the scheme $A$. Thus, the number of cycles broken by a given objects scheme $A$ increases when the size of $A$, i.e., the cardinality of its largest object, decreases.

Symmetrically, the number of local optima increases when the size of the objects scheme $A$ decreases. Indeed by Theorem 3.4, a free social outcome $z$ is a local optimum for a scheme $A$ if and only if for all social outcomes $x \succ_{\mathcal{R}} z \mathcal{H}_{x, z} \nsubseteq \mathcal{A}$, for all $\mathcal{A}$ belonging to the scheme $A$. It follows that if the objects scheme $A$ is composed by $k$ objects, then we have at least $(k-1)$ ! different local optima. Thus we obtain a decidability vs. non-manipulability trade-off. Schemes composed of a few large objects tend to produce cycles, while schemes of many small objects tend to produce considerably fewer cycles but increasingly many local optima. 
Remark 3.18. From what we have seen until now, it is obvious that, in order to work with the object construction power, it is helpful to find the hyperplanes involved in the fundamental cycles that generate all the others. In this respect, given an oriented graph $\mathcal{Y}_{\succeq_{\mathcal{R}}}$, it is helpful to consider a reduced homotopic one. Hence, for example, if $(x, y) \in Y_{1, \succeq_{\mathcal{R}}}$ is an edge given by a formal sum with coefficient 1 of edges which are in $Y_{1, \succeq_{\mathcal{R}}}$ then it can be deleted. So the graph in Figure 2.1 strongly simplifies in that in Figure $3.2^{6}$.

To be precise, the graph in Figure 3.2 does not exactly represent Salvetti's one, as the latter is in the complexification of $\mathcal{A}_{3,2}$, i.e., in $\mathbb{C}^{3} \simeq \mathbb{R}^{6}$, which allows paths to "go around" hyperplanes (e.g., the line and the arc joining 000 and 100 form, in the complex space, a loop around the hyperplane of equation $\lambda_{1}=0$ ). However Figure 3.2 gives a useful, though not totally precise, visual representation.

Looking at this figure we remark that it is not possible to retrieve the social rule from the graphic representation of the Salvetti graph: we do not know if agents prefer the social outcome 101 to 111 or viceversa; we only know that there is a cycle involving these two preferences. In order to get the precise information about two social outcomes we have to look at the rule.

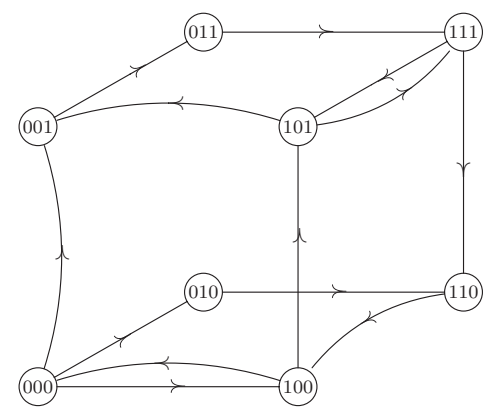

Figure 3.2. Reduced graph from Example 2.3.

Nevertheless the reduced graph carries very useful information. For example it is relatively simple to find the fundamental cycles that generate all the others. Looking at the graph in Figure 3.2 it is clear that, for this base, the fundamental cycles are:

$$
\begin{aligned}
& 001 \succ_{\mathcal{R}} 011 \succ_{\mathcal{R}} 111 \succ_{\mathcal{R}} 001, \\
& 000 \succ_{\mathcal{R}} 010 \succ_{\mathcal{R}} 110 \succ_{\mathcal{R}} 000, \\
& 000 \succ_{\mathcal{R}} 011 \succ_{\mathcal{R}} 111 \succ_{\mathcal{R}} 110 \succ_{\mathcal{R}} 000, \\
& 000 \succ_{\mathcal{R}} 100 \succ_{\mathcal{R}} 101 \succ_{\mathcal{R}} 110 \succ_{\mathcal{R}} 000 .
\end{aligned}
$$

By construction, the first one depends on the existence of the edge $(111,001)$, while the other three depend on $(110,000)$. This information allows us to determine which set of objects can break the cycles in the given social rule.

${ }^{6}$ For simplicity in the figure we omitted the hyperplanes, i.e., the coordinate hyperplanes of equations $\lambda_{i}=0$, for $i=1,2,3$. 
Clearly, there are many ways we can reduce the set $Y_{1, \succeq_{\mathcal{R}}}$ to a basic number of elements, and this number is not unique. However, all the reduced graphs represent exactly the same pairwise social preferences.

\subsection{On the median voter theorem}

In addition to intransitive cycles and agenda power, also another classic result of social choice theory, the median voter theorem, is subject to manipulability through object construction. By appropriately modifying the objects scheme, the outcome of a social decision may be made as distant as one wishes from the median voter's preferred social outcome.

Recall that the median voter theorem in its strong version [3,8] states that if a voter with median preferences exists, her/his most preferred outcome will beat any other alternative in pairwise majority voting. Indeed given a rule $\succ_{\mathcal{R}}$ with a median voter $m$, if $z$ is the opposite social outcome with respect to the median voter's preferred one and $z$ is free, then it is possible to find a scheme with preferred outcome $z$.

This is equivalent to saying that in our construction it is always possible to manipulate the objects scheme in such a way that the median voter theorem does not apply and social choice may converge to social outcomes very distant from the median voter's preferred one.

\section{Appendix}

\section{A. The Salvetti complex}

In Section 2 we described the cells of a complex. Obviously there is a boundary map on Salvetti's complex which is also very informative ${ }^{7}$. We will introduce the boundary only for the 1-skeleton of the complex $\mathcal{S}\left(\mathcal{A}_{n, m}\right)$.

Let us consider the free abelian groups $S_{1}\left(\mathcal{A}_{n, m}\right)$ and $S_{0}\left(\mathcal{A}_{n, m}\right)$ generated by $\mathcal{S}_{1}\left(\mathcal{A}_{n, m}\right)$ and $\mathcal{S}_{0}\left(\mathcal{A}_{n, m}\right)$ considering all the formal sums with integer coefficients.

The boundary map $\partial: S_{1}\left(\mathcal{A}_{n, m}\right) \longrightarrow S_{0}\left(\mathcal{A}_{n, m}\right)$ is defined as

$$
\partial\left(x_{i}, x_{j}\right)=x_{j}-x_{i}
$$

for any generator $\left(x_{i}, x_{j}\right) \in \mathcal{S}_{1}\left(\mathcal{A}_{n, m}\right)$, and the map extends to $S_{1}\left(\mathcal{A}_{n, m}\right)$ by linearity, i.e., if $c=\partial \sum_{j=1}^{k-1} a_{j}\left(x_{i_{j}}, x_{i_{j+1}}\right), a_{j} \in \mathbb{Z}$, is an element in $S_{1}\left(\mathcal{A}_{n, m}\right)$, then

$$
\partial c=\partial \sum_{j=1}^{k-1} a_{j}\left(x_{i_{j}}, x_{i_{j+1}}\right)=\sum_{j=1}^{k-1} a_{j} \partial\left(x_{i_{j}}, x_{i_{j+1}}\right) .
$$

7 The boundary map is a well know map in mathematics and it is a basic ingredient of Homology Theory. 
Given a social rule $\succeq_{\mathcal{R}}$ we obtain a graph $\mathcal{Y}_{\succeq_{\mathcal{R}}}$ which is, as we have seen in Section 2 , a subcomplex of Salvetti's complex in 0 and 1-dimensions. Then we can compute its boundary map. We may begin noticing that pairwise preferences correspond to formal sums with coefficients 1 in $Y_{1, \succ_{\mathcal{R}}}$ and that if two such formal sums are equivalent from a social point of view, i.e., they correspond to the same pairwise preferences, then they have the same boundary. Indeed, by linearity, the boundary of the sum in (2.1) is given by

$$
\begin{aligned}
\partial \sum_{j=1}^{k-1}\left(x_{j}, x_{j+1}\right) & =\sum_{j=1}^{k-1} \partial\left(x_{j}, x_{j+1}\right) \\
& =\sum_{j=1}^{k-1} x_{j+1}-\sum_{j=1}^{k-1} x_{j}=x_{k}-x_{1}=\partial\left(x_{1}, x_{k}\right),
\end{aligned}
$$

namely it depends only by its initial and final vertices.

Recall that a cycle $\gamma_{I} \in \mathcal{Y}$ is a subgraph with vertices $\left\{x_{1}, \ldots, x_{h}\right\}$ and edges $\left\{\left(x_{1}, x_{2}\right),\left(x_{2}, x_{3}\right), \ldots,\left(x_{h}, x_{1}\right)\right\}$. Hence the boundary $\partial \gamma_{I}$ of a cycle is equal to 0 . Indeed it is the formal sum with coefficient 1 of the edges in the subgraph $\gamma_{I}$ with same initial and final vertex $x_{1}$, i.e.

$$
\begin{aligned}
\partial \gamma_{I} & =\partial\left(x_{1}, x_{2}\right)+\partial\left(x_{2}, x_{3}\right)+\ldots+\partial\left(x_{h}, x_{1}\right) \\
& =x_{2}-x_{1}+x_{3}-x_{2}+\ldots+x_{1}-x_{h}=0 .
\end{aligned}
$$

Then to study the boundary map of a graph $\mathcal{Y}_{\succeq_{\mathcal{R}}}$ is equivalent to study the Condorcet-Arrow cycles of the corresponding social rule. The boundary operator transforms a geometric problem into an algebraic one and makes it possible to develop an algorithm that computationally performs the reduction of a given directed graph and allows to find cycles.

Example A.1. In Example 2.3 the boundary of each edge in $Y_{1, \succ_{\mathcal{R}}}$ is

$$
\partial\left(v_{1} v_{2} v_{3}, g_{1} g_{2} g_{3}\right)=g_{1} g_{2} g_{3}-v_{1} v_{2} v_{3} \text {. }
$$

We get a boundary matrix of 28 columns, one for each edge, and 8 rows, one for each vertex, with two entries equal 1 and -1 (those corresponding to the vertices of the edge in question) in each column, and all the other entries equal to 0 . Now our algorithmic implementation must simply delete any column which is the sum of two or more other columns. In our example, we can get the following reduced matrix

$$
\left[\begin{array}{ccccccccccc}
-1 & -1 & -1 & 1 & 0 & 0 & 0 & 0 & 0 & 0 & 0 \\
0 & 0 & 0 & 0 & -1 & 0 & 0 & 1 & 0 & 0 & 0 \\
0 & 1 & 0 & 0 & 0 & 0 & 0 & 0 & -1 & 0 & 0 \\
1 & 0 & 0 & 0 & 1 & -1 & 0 & 0 & 0 & 0 & 0 \\
0 & 0 & 1 & 0 & 0 & 0 & 0 & 0 & 0 & -1 & 0 \\
0 & 0 & 0 & 0 & 0 & 0 & 0 & 0 & 0 & 1 & -1 \\
0 & 0 & 0 & -1 & 0 & 0 & 1 & 0 & 1 & 0 & 1 \\
0 & 0 & 0 & 0 & 0 & 1 & -1 & -1 & 0 & 0 & 0
\end{array}\right]
$$


where the columns are ordered as

$$
\begin{aligned}
& \{(000,011),(000,010),(000,100),(110,000),(001,011),(011,111), \\
& (111,110),(111,001),(010,110),(100,101),(101,110)\}
\end{aligned}
$$

and the rows are ordered lexicographically. If the sum, with coefficient 1 , of three or more columns is the zero column, then we have a cycle. Indeed, for example, the sum of the 5-th 6-th and 8-th columns is 0 and it corresponds to the first cycle described in (3.8). Hence from the above matrix it is computational easy to retrieve all cycles.

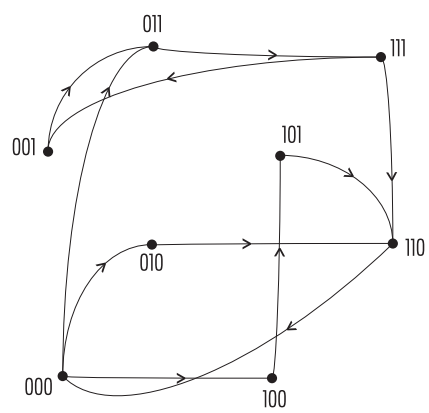

Figure A.1. Reduced graph with a base of 11 elements.

Moreover we get that the graph in Figure 2.1 can be reduced to that in Figure A.1 where cycles are easier to be noticed.

\section{References}

[1] G. Amendola and S. Settepanella, Modularity and optimality in social choice, J. Math. Sociol. 36 (2012), 44-77.

[2] Y. M. BARYSHNIKOV, Topological and discrete social choice: in a search of a theory, Social Choice Welf. 14 (1997), 199-209.

[3] D. BLACK, "The Theory of Committees and Elections", Cambridge University Press, Cambridge, 1958.

[4] G. Chichilnisky, Social choice and topology of spaces of preferences, Adv. Math. 37 (1980), 165-176.

[5] G. CHICHILNISKY, Social choice and game theory: recent results with a topological approach, In: "Social Choice and Welfare", P. K. Pattanaik and M. Salles (eds.), North Holland, Amsterdam, 1983, 79-102.

[6] J.-A.-N. Condorcet de Caritat Marquis de, "Essai sur l'Application de l'Analyse aux Probabilités de Decision Rendue à la Pluralité des Voix", Imprimerie Royale, 1785, Paris.

[7] A. T. Denzau and R. J. Mackay, Structure-induced equilibria and perfect-foresight expectations, Amer. J. Political Science 25 (1981), 762-779.

[8] A. Downs, An economic theory of political action, J. Political Economy 65 (1957), 135150.

[9] B. EcKMann, Räume mit Mittelbildungen, Comment. Math. Helv. 28 (1954), 329-340. 
[10] B. Eckmann, T. Ganea and P. J. Hilton, Generalized means, In: "Studies in Mathematical Analysis and Related Topics. Essays in honor of George Pólya", G. Szegö (ed.) Stanford University Press, Stanford, 1962, 82-92.

[11] J. M. ENELOW and M. J. HINICH, Voting one issue at a time: The question of voter forecasts, Amer. Political Science Rev. 77 (1983), 435-445.

[12] R. FRYER and M. JACKSON, A Categorical model of cognition and biased decision making, B.E. J. Theor. Econ. 8 (2008), Article 6.

[13] G. H. KRAMER, Sophisticated voting over multidimensional choice spaces, J. Math. Sociol. 2 (1972), 165-180.

[14] L. Marengo and C. Pasquali, The construction of choice. A computational voting model, discussion paper, LEM, Scuola Superiore Sant'Anna, Pisa, WP 2008/24.

[15] W. S. MASSEY, "A Basic Course in Algebraic Topology”, Springer Verlag, Berlin, 1981.

[16] R. MCKELVEY, Intransitivities in multi-dimensional voting models and some implications for agenda control, J. Econom. Theory 16 (1976), 472-482.

[17] S. Mullainathan, Thinking through Categories, MIT working paper.

[18] S. Mullainathan, J. Schwartzstein and Andrei Shleifer, Coarse thinking and persuasion, Q. J. Econom. 123 (2008), 577-619.

[19] C. R. Plott and M. E. Levine, A model of agenda influence on committee decisions, American Econom. Rev. 68 (1978), 146-160.

[20] D. S AARI, "Geometry of Voting", Springer Verlag, New York, 1994.

[21] D. G. SAARI, Mathematical structure of voting paradoxes 1: Pairwise vote, Econom. Theory $15(2000), 1-53$.

[22] D.G. SAARI, Mathematical structure of voting paradoxes 2: Positional voting, Econom. Theory, 15 (2000), 55-101.

[23] M. S ALVETTI, Topology of the complement of real hyperplanes in $\mathbb{C}^{N}$, Invent. Math. 88 (1987), 603-618.

[24] S. SetTePanella, Social choice among complex objects: Mathematical tools, LEM Working Paper Series, WP 2010/1.

[25] K. A. SHEPSLE, Institutional arrangements and equilibrium in multidimensional voting models, Amer. J. Political Science 23 (1979), 27-59.

[26] H. A. Simon, "The Sciences of the Artificial", 2nd ed., MIT Press, Cambridge, MA, 1982.

[27] H. TERAO, Chambers of arrangements of hyperplanes and arrow's impossibility theorem, Adv. Math. 214 (2007), 366-378.

[28] S. WeINBERGER, On the topological social choice model, J. Econom. Theory 115 (2004), 377-384.

Department of Business and Management LUISS

Viale Romania, 32

00197 Roma, Italia

1.marengo@luiss.it

Department of Mathematics

Hokkaido University

Sapporo, 060-0810, Japan

s.settepanella@math.sci.hokudai.ac.jp 